\title{
Recalescence after solidification in Ge films melted by picosecond laser pulses
}

\author{
J. Siegel, ${ }^{\text {a) }}$ J. Solis, and C. N. Afonso \\ Instituto de Optica, CSIC, C/Serrano 121, 28006 Madrid, Spain
}

(Received 30 April 1999; accepted for publication 30 June 1999)

\begin{abstract}
Thin amorphous Ge films on glass substrates are irradiated by single picosecond (ps) laser pulses and the induced melting and solidification process is followed by means of real-time reflectivity measurements with ps resolution using a setup based on a streak camera. Due to the excellent time resolution achieved in single exposure, the recalescence process occurring upon solidification can be completely resolved by means of an all-optical technique. The results are consistent with the bulk nucleation of the amorphous phase in the supercooled liquid at an extremely large nucleation rate. The massive release of solidification heat causes the reheating and partial remelting of the film after its complete solidification. The occurrence of recalescence after solidification is responsible for the formation of the crystalline phase finally obtained. (c) 1999 American Institute of Physics. [S0003-6951(99)03334-3]
\end{abstract}

Pulsed-laser melting of semiconductors may lead to a variety of different solidification mechanisms. ${ }^{1-11}$ In this context, the importance of recalescence phenomena, i.e., the reheating of the material due to the release of latent heat during solidification, has already been pointed out by several authors. ${ }^{3,6,7,9,10,11}$ In a comprehensive work, Sameshima and Usui $^{7}$ demonstrated that recalescence effects can be reduced or enhanced by a proper variation of the film thickness and the thermal conductivity of the substrate. But only by means of real-time reflectivity, $6,10,11$ (RTR) and real-time conductance $^{6,7}$ (RTC) measurements the dynamics of recalescence phenomena could be studied. All authors appear to agree on a model which assumes that recalescence effects take place during the solidification process, thus slowing it down and favoring the formation of the crystalline phase. The time resolution of those techniques is several nanoseconds (ns), which is generally considered to be sufficient to follow the main features of the solidification processes. Nevertheless, solidification processes might occur much faster than the duration of the ns laser pulses ${ }^{7}$ used in most of the experiments reported, and thus, their complete understanding might require sub-ns resolution measurements.

In this letter, we provide direct evidence for the occurrence of massive recalescence after the complete solidification of the material. This has been possible by means of RTR measurements with picosecond (ps) resolution. The excellent time resolution achieved also enables us to determine the high nucleation rate at the early stage of solidification. The results obtained imply that the amorphous phase is nucleated directly from the supercooled melt through a bulk solidification process. The occurrence of massive recalescence is found to catalyze the subsequent amorphous-to-crystalline transformation.

The material system under study is formed by a $50-\mathrm{nm}-$ thick amorphous Ge film grown by dc sputtering on a glass substrate. As-grown areas of the specimens were irradiated

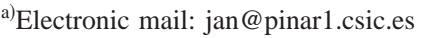

by single $30 \mathrm{ps}$ laser pulses at $583 \mathrm{~nm}$. The dynamics of the induced transformations has been followed in real time by measuring the reflectivity changes at the air/film interface by means of a probe laser beam incident at a small angle $\left(7^{\circ}\right)$ and focused at the center of the irradiated area. The probe beam used was provided either by a $\mathrm{He}-\mathrm{Ne}$ laser $(633 \mathrm{~nm})$ or by a single-mode $\mathrm{Ar}^{+}$laser $(514 \mathrm{~nm})$. The detection system used was either a fast photodetector connected to a transient digitizer for RTR measurements with ns resolution or a streak camera for RTR measurements with ps resolution. More details about the experimental setup can be found elsewhere. ${ }^{11}$ Unlike the common experimental setup based on a pump-and-probe scheme, ${ }^{12}$ our streak camera-based setup permits the measurement of ultrafast processes upon single laser pulse exposure.

Figure 1 shows a RTR transient recorded at $514 \mathrm{~nm}$ upon irradiation with a single laser pulse at a fluence of 65 $\mathrm{mJ} / \mathrm{cm}^{2}$. The actual duration of the laser pulse itself (30 ps) can be resolved in an entire time window of $1 \mathrm{~ns}$, which confirms the excellent time resolution of the experimental setup. The induced increase of the reflectivity by $\approx 50 \%$ is consistent with the melting of the material surface. ${ }^{11,13}$ It is remarkable that the reflectivity rise, and thus, the melting process, occurs within the pulse duration as predicted by several authors. ${ }^{14,15}$ Similar results (fs laser pulse melting of

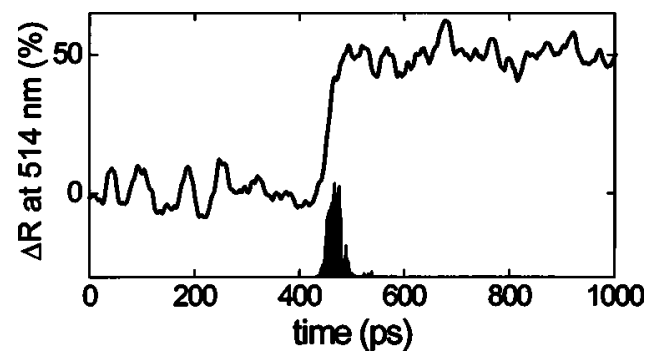

FIG. 1. Transient reflectivity increase at $514 \mathrm{~nm}$ upon melting of the $a$-Ge film by a single ps laser pulse $\left(65 \mathrm{~mJ} / \mathrm{cm}^{2}\right)$ as measured by a streak camera in a time window of $1 \mathrm{~ns}$. The temporal profile of the laser pulse (full width at half maximum $=30 \mathrm{ps}$ ) is included at the bottom of the figure. 


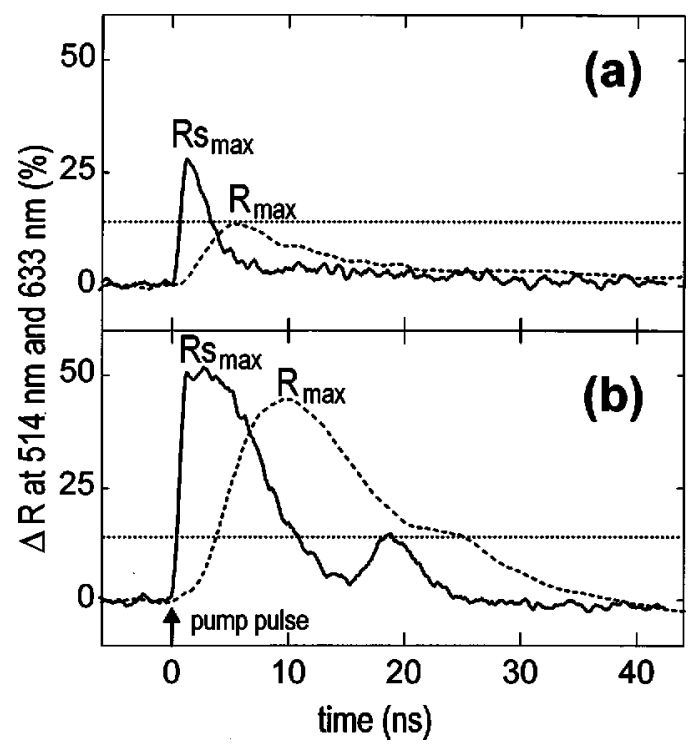

FIG. 2. Comparison between reflectivity transients at (a) $19 \mathrm{~mJ} / \mathrm{cm}^{2}$ and (b) $65 \mathrm{~mJ} / \mathrm{cm}^{2}$ measured at $633 \mathrm{~nm}$ with $\mathrm{ns}$ resolution (dashed curves) and at $514 \mathrm{~nm}$ with ps resolution (solid curves). The dotted lines indicate the reflectivity change predicted for $a$-Ge at its melting temperature.

Si within a few ps) have already been reported by timeresolved pump-and-probe measurements. ${ }^{12}$ Nevertheless, such a ps melting process has not been measured before in real time in a single exposure experiment.

In order to compare the present ps-resolved measurements with the earlier results obtained with ns resolution, ${ }^{11}$ we have performed simultaneously RTR measurements with both ns and ps resolution. Figures 2(a) and 2(b) show representative transients at two different fluences in the same time window of $50 \mathrm{~ns}$. It is evident that neither the melting nor the solidification process can be completely resolved with nsresolution measurements. The poor time resolution affects also the maximum values $R_{\max }$ of the RTR transients measured with ns resolution, which are smaller than the actual $R s_{\max }$ values obtained by ps-resolution measurements. This drawback of the ns-resolution measurements can be partly compensated by additional RTR measurements at the film/ substrate interface. ${ }^{11}$ Nevertheless, this requires the use of a transparent substrate, a thin film, and the realization of two sets of measurements. The measurements with ps resolution are not only free from these limitations but also capable of resolving precisely events occurring in a much shorter time scale. For instance, the so-measured melt threshold (15 $\mathrm{mJ} / \mathrm{cm}^{2}$ ) is clearly below the one provided by the nsresolution measurements ${ }^{11}\left(20 \mathrm{~mJ} / \mathrm{cm}^{2}\right)$. As a common criterion to define the melt threshold, we have used the fact that the reflectivity of $a-\mathrm{Ge}$ at the melting temperature is $14 \%$ higher than the value at room temperature. ${ }^{11}$

The solidification process indeed occurs mainly in the ns time scale, which permits, even by means of ns-resolution measurements, ${ }^{11}$ estimating the solidification time and the observation of film recalescence at high fluences [the shoulder in Fig. 2(b)]. Nevertheless, substantial differences in the dynamics of recalescence can be observed when measured with ps resolution. What has seemed to be a shoulder in the ns-resolution RTR transient, and thus a slowdown of the solidification process, appears as a clear second maximum in the ps-resolution RTR transient [Fig. 2(b)]. The dip between Downloaded 09 Mar 2010 to 161.111.180.191. Redistribution subje

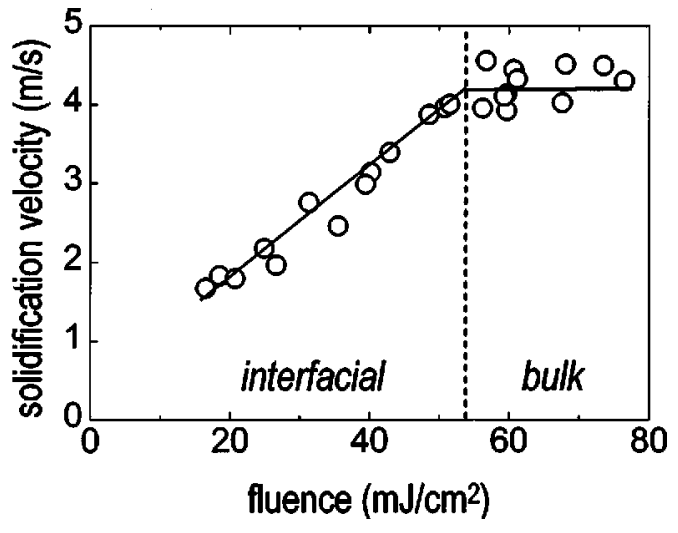

FIG. 3. Solidification velocity of the $a$-Ge film as a function of laser fluence as obtained by streak camera measurements. The solid lines are a guide to the eye. The dashed vertical line marks the onset of recalescence and crystallization.

the two maxima is well below the $14 \%$ level, which corresponds to the reflectivity of the amorphous solid film at the melting temperature, ${ }^{11}$ and thus evidences that the film has solidified completely before massive recalescence occurs. Such a recalescence process taking place after the complete solidification of the material has not earlier been reported in the literature. Whether this fact is a consequence of the use of ps laser pulses or a general feature of the solidification dynamics in thin semiconductor films, and thus occurring also under ns laser pulse irradiation, cannot be decided at this point. Nevertheless, it is clear that measurements with sub-ns resolution are also essential in ns laser pulse irradiation experiments in order to study in detail the recalescence process.

A very detailed description of the entire melting and solidification process at high fluences can be obtained following the ps-resolution transient shown in Fig. 2(b). Melting occurs within the pulse duration, and during the following few ns the molten volume cools down by heat conduction to the substrate, leading to a highly supercooled liquid. Due to the high thermal conductivity of liquid $\mathrm{Ge}$, the liquid reaches an internal thermal gradient as low as 0.01 $\mathrm{K} / \mathrm{nm}$ during the cooling process, as reported elsewhere. ${ }^{7}$ Then, massive nucleation of the solid phase throughout the supercooled liquid initiates solidification, which leads to a fast decrease of the reflectivity to a level consistent with a completely solidified, although still hot material. The release of the solidification enthalpy then becomes dominant, leading to an increase of the temperature to the melting point $(\Delta R=14 \%)$. This recalescence process, which involves partial remelting of the solid phase obtaining a state of reduced supercooling, promotes the formation of the crystalline phase. The final state of the material consists of a mixture of amorphous and crystalline phases with a maximum crystalline fraction of $\approx 60 \%$, as shown in Ref. 11 .

At low fluences [Fig. 2(a)], solidification occurs interfacially leading to the reamorphization of the film, as demonstrated earlier. ${ }^{11}$ This result can be quantified with the help of the ps-resolution measurements by determining the solidification times $t_{s}$ and the initial melt depths $z_{s}$ with high precision. The $t_{s}$ values are defined as the time during which the reflectivity remains $14 \%$ above the initial value ${ }^{11}$ and the $z_{s}$ values can directly be deduced from the $R s_{\max }$ values with the help of optical calculations and following the description to AIP license or copyright; see http://apl.aip.org/apl/copyright.jsp 
in Ref. 11. From these data, the solidification velocities $\nu_{s}$ $=z_{s} / t_{s}$ have been calculated and plotted in Fig. 3 as a function of fluence. Their evolution shows clearly that there are two solidification regimes separated by the recalescence threshold at $54 \mathrm{~mJ} / \mathrm{cm}^{2}$, which can distinctly be appreciated by a change in the trend of $\nu_{s}$ : In the interfacial reamorphization regime at low fluences, $\nu_{s}$ increases linearly from 1.7 to $4 \mathrm{~m} / \mathrm{s}$. In the high fluence regime, where partial crystallization occurs via bulk solidification, and thus, no solidification front propagates, the behavior of $\nu_{s}$ remains apparently constant. The continuous transition of the behavior of $\nu_{s}$ from the low to the high fluence regime clearly indicates that also in the latter regime solidification is initiated by the nucleation of the amorphous phase, which then is partially transformed into the crystalline phase through recalescence. This interpretation is further supported by our observation that the transient shape changes only by the appearance of the second maximum when increasing the fluence above the recalescence threshold. The presence of a still considerable amount of amorphous material (40\%) in the finally solidified film also indicates that the amount of latent heat released during the initial nucleation and growth process of the amorphous phase is not sufficient to promote the crystallization of the entire film.

In the case of bulk solidification, an estimation of the minimum nucleation rate in the supercooled liquid can be made following the calculations of Sameshima and Usui. ${ }^{7}$ Assuming that the solid nuclei grow spherically with a diameter $d$, a minimum value for the nucleation density is given by the inverse of the sphere volume $\left[4 \pi(d / 2)^{3} / 3\right]$. Taking into account the fact that the mean value of the crystal size has been determined ${ }^{16}$ to be $\approx 10 \mathrm{~nm}$, a minimum nucleation density of $1.9 \times 10^{24}$ events $/ \mathrm{m}^{3}$ is obtained. Dividing this value by the mean value of the solidification time, a minimum value of $1.8 \times 10^{32}$ events $/ \mathrm{m}^{3} \mathrm{~s}$ for the nucleation rate is obtained. This value is more than one order of magnitude above those earlier reported for rapid solidification in elementary semiconductors. This indicates that the degree of supercooling prior to bulk solidification has to be higher than the $530 \mathrm{~K}$ value reported by Stiffler, Thompson, and Peercy ${ }^{6}$ upon ns laser pulse irradiation of 300-nm-thick $c$-Ge films on a $\mathrm{SiO}_{2}$ substrate. The same authors found evidence for solidification being initiated by nucleation of the amorphous phase, which subsequently transformed to crystalline, cata- lyzed by recalescence. Our results are thus in good agreement with their observations, although they could not observe a temporal separation between the solidification and the recalescence process, either due to the limited time resolution or the larger thickness of the films they used.

In summary, RTR measurements with ps resolution in single exposure improve considerably our understanding of pulsed-laser melting and solidification processes in semiconductors and reveal exciting features. Ge films are found to solidify completely before recalescence takes place, catalyzing the amorphous-to-crystalline transformation. The supercooling prior to solid-phase nucleation and the minimum nucleation rate are $>530 \mathrm{~K}$ and $2 \times 10^{32}$ events $/ \mathrm{m}^{3} \mathrm{~s}$, respectively. The interface velocity of the reamorphization front at low fluences could be determined precisely, showing a linearly increasing behavior with fluence from 1.7 to $4 \mathrm{~m} / \mathrm{s}$ up to the recalescence threshold at $54 \mathrm{~mJ} / \mathrm{cm}^{2}$.

This work has been partially supported by CICYT (Spain) under Project No. TIC93-0125. One of the authors (J.S.) acknowledges with pleasure the funding of the European Community through a Marie Curie Fellowship (ERB40001GT954352) within the TMR Program.

${ }^{1}$ Laser Annealing of Semiconductors, edited by J. M. Poate and J. W. Mayer (Academic, New York, 1982), and references cited therein.

${ }^{2}$ M. O. Thompson, J. W. Mayer, A. G. Cullis, H. C. Webber, N. G. Chew, J. M. Poate, and D. C. Jacobson, Phys. Rev. Lett. 50, 896 (1983).

${ }^{3}$ R. F. Wood and G. A. Geist, Phys. Rev. Lett. 57, 873 (1986).

${ }^{4}$ D. H. Lowndes, G. E. Jellison, Jr., S. J. Pennycook, S-P. Withrow, and D. N. Mashburn, Appl. Phys. Lett. 48, 1389 (1986).

${ }^{5}$ J. J. P. Bruines, R. P. M. van Hal, H. M. J. Boots, W. Sinke, and F. W. Saris, Appl. Phys. Lett. 48, 1252 (1986).

${ }^{6}$ S. R. Stiffler, M. O. Thompson, and P. S. Peercy, Appl. Phys. Lett. 56, 1025 (1990).

${ }^{7}$ T. Sameshima and S. Usui, J. Appl. Phys. 74, 6592 (1993).

${ }^{8}$ J. S. Im, H. J. Kim, and M. O. Thompson, Appl. Phys. Lett. 63, 1969 (1993).

${ }^{9}$ F. Vega, C. N. Afonso, W. Szyszko, and J. Solis, J. Appl. Phys. 82, 1 (1997).

${ }^{10}$ J. Boneberg and P. Leiderer, Phys. Status Solidi A 166, 643 (1998).

${ }^{11}$ J. Siegel, J. Solis, and C. N. Afonso, J. Appl. Phys. 84, 5531 (1998).

${ }^{12}$ See, for instance, C. V. Shank, R. Yen, and C. Hirlimann, Phys. Rev. Lett. 50, 454 (1983).

${ }^{13}$ G. E. Jellison and D. H. Lowndes, Appl. Phys. Lett. 51, 352 (1987).

${ }^{14}$ D. Agassi, J. Appl. Phys. 55, 4376 (1984).

${ }^{15}$ N. Bloembergen, Mater. Res. Soc. Symp. Proc. 51, 3 (1986).

${ }^{16}$ J. Solis, J. Siegel, C. Garcia, J. Jimenez, and R. Serna, Mater. Res. Soc. Symp. Proc. 452, 839 (1997). 